\title{
Body mass index and adipokines/cytokines dysregulation in Systemic Sclerosis
}

\author{
F Iannone ${ }^{1}$, Emanuela Praino ${ }^{1}$, Cinzia Rotondo ${ }^{1}$, Dorotea Natuzzi ${ }^{2}$, Rita Bizzoca ${ }^{2}$, Nunzia \\ Lacarpia $^{2}$, Marco Fornaro ${ }^{1}$, and Fabio Cacciapaglia ${ }^{2}$ \\ ${ }^{1}$ Rheumatology Unit \\ ${ }^{2}$ Rheumatolgy Unit
}

April 7, 2021

\begin{abstract}
Body fat has regulatory functions through producing cytokines and adipokines whose role in the pathogenesis of Systemic Sclerosis (SSc) is currently emerging. Changes in body mass, either overweight or underweight status, entail a dysregulation of the cytokines/adipokines network that may impact on SSc disease activity. We evaluated serum levels of adipokines and cytokines in SSc patients and correlated them to clinical features and body mass index (BMI) categories. The study included $89 \mathrm{SSc}$ patients and 26 healthy donors (HD). Serum levels of adiponectin, leptin, resistin, visfatin, TNF $\alpha$, IFN $\gamma$, IL-2, IL-10, and IL-17A were measured by Multiplex Immunoassay, and correlated to BMI, waist to hip ratio, and disease specific features. Mann-Whitney U-test or t-Student for unpaired data, Kruskal-Wallis test or ANOVA, were used for comparisons between groups. Spearman's or Pearson's test were used for correlation analysis. Serum levels of TNF $\alpha$, IL-2, leptin, and resistin, were significantly higher in SSc than in HD. The highest levels of IL-17A, IL-2, IL-10, leptin and visfatin were detected in obese SSc patients $(\mathrm{p}<0.01)$. Conversely, underweight SSc patients showed the highest TNF $\alpha$ levels $(\mathrm{p}<0.05)$, which were negatively correlated with BMI $(\mathrm{p}=0.05)$. No correlation between adipokines/cytokines and clinical characteristics was found. Adipokines, IL-2, IL-10 and IL-17A were found to be increased in obese SSc patients, but whether they play a role in the pathogenesis of the disease remains to be investigated. Intriguingly, underweight patients had higher TNF $\alpha$ levels, suggesting a potential role of $\mathrm{TNF} \alpha$ in inducing the cachexia observed in long-lasting disease.
\end{abstract}

Title: Body mass index and adipokines/cytokines dysregulation in Systemic Sclerosis Running Title: Systemic sclerosis, adipokines and BMI

Florenzo Iannone*, Emanuela Praino*, Cinzia Rotondo, Dorotea Natuzzi, Rita Bizzoca, Nunzia Lacarpia, Fabio Cacciapaglia.

*These authors equally contributed to this work

${ }^{1}$ Rheumatology Unit, Department of Emergency and Transplantation, University of Bari, Bari Italy

${ }^{2}$ Department of medical and surgical sciences - Rheumatology Unit, University of Foggia, Italy

${ }^{1}$ Florenzo Iannone

Full Professor of Rheumatology, MD, PhD florenzo.iannone@uniba.it

${ }^{1}$ Emanuela Praino

Research Assistant, MD emanuelapraino@yahoo.it

${ }^{2}$ Rotondo Cinzia 
Research Assistant, MD cinzia.rotondo@gmail.com

${ }^{1}$ Dorotea Natuzzi

Biologist, BD dorotea.natuzzi@uniba.it

${ }^{1}$ Rita Bizzoca

Biologist, BD rita.bizzoca@uniba.it

${ }^{1}$ Nunzia Lacarpia

Biologist, BDnunzia.lacarpia@uniba.it

${ }^{1}$ Marco Fornaro

Research Assistant, MD marco3987@hotmail.it

${ }^{1}$ Fabio Cacciapaglia

Lecturer, MD, PhD fabio.cacciapaglia79@gmail.com

Corresponding author: Florenzo Iannone, MD, PhD, Full Professor of Rheumatology

Rheumatology Unit, Department of Emergency and Transplantation, Policlinico, Piazza G. Cesare 11, 70124

Bari, Italy. E-mail:florenzo.iannone@uniba.it

Key words: Visfatin, Leptin, Adiponectin, body weight

Competing interest: none

Funding: the study had no financial support.

Number of words:

\section{ABSTRACT}

Body fat has regulatory functions through producing cytokines and adipokines whose role in the pathogenesis of Systemic Sclerosis (SSc) is currently emerging. Changes in body mass, either overweight or underweight status, entail a dysregulation of the cytokines/adipokines network that may impact on SSc disease activity. We evaluated serum levels of adipokines and cytokines in SSc patients and correlated them to clinical features and body mass index (BMI) categories.

The study included 89 SSc patients and 26 healthy donors (HD). Serum levels of adiponectin, leptin, resistin, visfatin, $\mathrm{TNF} \alpha, \mathrm{IFN} \gamma, \mathrm{IL}-2$, IL-10, and IL-17A were measured by Multiplex Immunoassay, and correlated to BMI, waist to hip ratio, and disease specific features. Mann-Whitney U-test or t-Student for unpaired data, Kruskal-Wallis test or ANOVA, were used for comparisons between groups. Spearman's or Pearson's test were used for correlation analysis.

Serum levels of TNF $\alpha$, IL-2, leptin, and resistin, were significantly higher in SSc than in HD. The highest levels of IL-17A, IL-2, IL-10, leptin and visfatin were detected in obese SSc patients $(p<0.01)$. Conversely, underweight SSc patients showed the highest TNF $\alpha$ levels $(p<0.05)$, which were negatively correlated with BMI $(p=0.05)$. No correlation between adipokines/cytokines and clinical characteristics was found.

Adipokines, IL-2, IL-10 and IL-17A were found to be increased in obese SSc patients, but whether they play a role in the pathogenesis of the disease remains to be investigated. Intriguingly, underweight patients had higher $\mathrm{TNF} \alpha$ levels, suggesting a potential role of $\mathrm{TNF} \alpha$ in inducing the cachexia observed in long-lasting disease.

\section{Introduction}

Systemic sclerosis (SSc) is a connective tissue disease characterized by small vessels vasculopathy associated with fibrosis of multiple organs. The pathogenesis is unclear, but an autoimmune dysregulation and an 
abnormal inflammatory response seem to be involved in the early stage of the disease. Emerging evidence suggests that white adipose tissue (WAT), besides having the role of energy storage, is now acknowledged as a pleiotropic organ with endocrine functions and regulating immune and inflammatory responses, being a source of cytokines and adipokines (leptin, adiponectin, visfatin, resistin) (1). Indeed, obesity may have an impact on disease activity as well as on clinical response of patients with rheumatoid arthritis and psoriatic arthritis $(2,3)$. In obese subjects, leptin induces the expression of adhesion molecules on endothelial cells and activates macrophages, while hindering adiponectin production by adipocytes, contributing to the "low-grade inflammatory state" associated with obesity (1).

Recently, the role of cytokines/adipokines in the pathophysiology of SSc has become a matter of investigation but studies correlating them with the clinical subsets or particular organ involvement were not always consistent. Serum leptin levels were found to be positively correlated with body mass index (BMI), negatively with disease activity in SSc patients, but not increased in comparison with healthy controls $(4,5)$. Conversely, serum levels of leptin, resistin and TNF $\alpha$ were higher in a small cohort of 16 SSc patients than in control subjects but no correlation with skin involvement, disease duration and disease activity was seen (6). A newly discovered adipokine, adipsin, was significantly higher in limited cutaneous SSc (lcSSc) than in diffuse cutaneous SSc (dcSSc) and was strongly associated with pulmonary arterial hypertension (7). Interestingly, adiponectin seems to play a protective role in SSc, as the levels were found to be low in dcSSc patients and inversely correlated with the extension of skin fibrosis, C-reactive protein (CRP) and erythrocyte sedimentation rate (ESR) (5,8-10). However, the interweave among body mass, cytokines/adipokines and SSc clinical phenotypes has been poorly investigated.

In this study, we aimed at evaluating serum levels of adipokines (leptin, resistin, visfatin, adiponectin) and cytokines ( $\mathrm{TNF} \alpha$, Interferon-gamma (IFN $\gamma$ ), IL-2, IL-10, IL-17A) in SSc patients according to BMI categories and disease specific characteristics.

\section{Materials \& Methods}

\section{Ethical approval}

The study obtained the approval of the local ethics committee (Azienda Policlinico Bari, n.5351 14/09/2017). The study was conducted in compliance with the Helsinki's declaration, and all patients gave their written informed consent to participate and for use of their data for publication, with explicit protection of their identity

\section{Patient group}

We evaluated 89 Caucasian patients with Systemic Sclerosis (SSc) fulfilling the 2013 ACR/ EULAR classification criteria (11) and 26 healthy blood donors (HD) as control group, including subjects not suffering from any disease and not taking medications at enrolment.

\section{Cytokine assay}

Blood samples obtained from all participants were centrifuged and serum was separated and stored in aliquots at $-80{ }^{\circ} \mathrm{C}$ until use. Serum levels of adipokines (adiponectin, leptin, resistin, visfatin) and cytokines (TNF $\alpha$, IFN $\gamma$, IL-2, IL-10, IL-17A) were measured, in duplicate, using kits manufactured by Bio-Rad (Bioplex ProTM Cytokine / Chemokine and Growth Factor Assay and Bioplex Pro Diabetes Assay) for Multiplex Immunoassay (Bioplex 200 System by Bio-Rad Laboratories, Hercules (CA, USA). The analyses were carried out according to the manufacturer's instructions.

\section{Clinical data}

In all patients we collected clinical, anthropometric and laboratory features, including disease duration, onset of Raynaud's phenomenon, skin involvement according to the modified Rodnan Skin Score (mRSS) (12) and cutaneous subset according to LeRoy criteria (13). Moreover, the presence/absence of interstitial lung disease (ILD) defined by chest HR-CT scan, pulmonary function test (PFT) with FVC, FEV1/FVC, DLCO and RV estimation, the 6-minute walking distance test (6mWDT), the presence/absence of pulmonary 
arterial hypertension (PAH) diagnosed by right heart catheterization, the presence/absence of esophagopathy evaluated at esophageal scintigraphy or esophageal manometry or chest HR-CT scan, the presence of digital pitting-scars and/or digital ulcers (past or active), the nailfold capillaroscopic pattern "Early", "Active" and "Late" (14), the disease activity according to the ESSG (European Scleroderma Study Group) disease activity index (15), were recorded. Drugs investigated were: peripheral vasodilators, iloprost, immunosuppressive drugs, glucocorticoids, antihypertensive and lipid-lowering agents, targeted therapy for the treatment of PAH (bosentan, sildenafil, tadalafil, ambrisentan), oxygen therapy. The BMI, waist circumference (Waist C) and Waist-to-Hip Ratio (WHR), cardiovascular risk index, acute phase proteins, cholesterol and triglycerides serum levels, previous smoking status, comorbidity (such as arterial hypertension, diabetes mellitus and hyperlipidemia) were also assessed. The BMI was calculated as weight in kilograms divided by the height in square meters and in accordance with the WHO BMI category classification, patients were considered underweight (BMI <18.5), normal-weight (BMI=18.5-24.99), overweight (BMI=25-29.99) and obese ([?]30). The cardiovascular (CV) risk was estimated as the ratio of total cholesterol/high-density-lipoprotein (HDL), or applying the Framingham Risk Score (FRS).

\section{Statistical Analysis}

Data were analyzed using IBM-SPSS Statistics 20 software. The comparison between two groups were done by Mann-Whitney U-test or t-Student, or chi-square test where appropriate. Comparisons among several groups were made using Kruskal-Wallis test or ANOVA, as appropriate. Spearman's or Pearson's test were used for correlations between cytokine/adipokines and clinical characteristics. Multiple linear regressions were performed for the analysis of predictors. A p-value [?] 0.05 was considered statistically significant.

\section{Results}

\section{Patient characteristics}

Clinical characteristics of SSs patients are shown in Table 1. Most patients had limited cutaneous SSc (83\%), and $27 \%$ had active disease ([?] 3 ESSG score). Of note, only $5(6 \%)$ patients were obese (BMI [?] 30), while 9 (10\%) were underweight (BMI [?] 18.5). At study entry, drugs taken were: calcium channel blockers (CCB) $(67.4 \%)$, iloprost (48.3\%) glucocorticoids (34.8\%), endothelin receptor antagonists (ERA) (22.3\%), methotrexate (19.5\%), mycophenolate mofetil (13.5\%), phosphodiesterase-5 inhibitors (PDE5i) (tadalafil, sildefanil) (8.9\%). No patient was taking non-steroidal anti-inflammatory drugs. In Table 2, the comparison of demographics, anthropometric characteristics, and serum levels of cytokines and adipokines between SSc and HD are shown. No significant differences in anthropometric characteristics were detected, but a trend toward a lower BMI was seen in SSc patients.

\section{Adipokines and Cytokines}

Table 2 shows the comparison of cytokines and adipokines between HD and the whole SSc cohort. Leptin and resistin were significantly higher in SSc than in HD. Visfatin was also increased in SSc, but the difference from HD did not reach the statistically significance. Adiponectin was measured only in the SSc group (see below). Remarkably, SSc patients showed significantly higher levels of TNF $\alpha$ and IL-2 than HD. Because of a possible bias by drugs, serum levels of cytokines and adipokines were correlated to the different treatments by multiple linear regression models. To this end, patients were grouped into SSc-glucocorticoids, SSc-ERA, SSc-immunosuppressants, SSc-CCB, SSc-iloprost, SSc-PDE5i. Only PDE5 inhibitors were independently associated with higher levels of leptin (OR 3.27, 95\% CI 12892-52716, $\mathrm{p}=0.002$ ) and visfatin (OR 2.66, 95\% CI 4340-29950, $\mathrm{p}=0.009$ ). Therefore, SSc patients were stratified into those taking PDE5i and those who did not, and compared by cytokines and adipokines levels (Table 2). Although only 8 SSc patients were on treatment with PDE5i, their levels of leptin and visfatin were significantly higher than in SSc patients without PDE5i (Table 2), while no difference was detected of the remaining cytokines.

No statistically significant difference in cytokines/adipokines levels between early SSc $(<2$ years of disease duration) and late disease ( $>2$ years of disease duration) was observed. We also stratified SSc patients as high and low CV risk according to the Framingham score and other indices (cholesterol /HDL ratio $>4.5$, waist 
circumference $>88 \mathrm{~cm}$, WHR $>0.8$ ). As only 3 patients had high/medium CV FRS, statistical comparison with those showing low risk CV FRS could not be made (data not shown). Furthermore, no significant differences were found when patients were stratified according to DLCO $<75 \%$ or FVC $<70 \%$ values, or the disease activity index ESSG $<3$. Likewise, no peculiar pattern of cytokines/adipokines was found in lcSSc or dcSSc subsets.

\section{BMI categories}

When SSc patients were subdivided by BMI categories, some meaningful differences emerged (Table 3). As expected, leptin (Figure 1) and visfatin levels were significantly higher in obese than in other BMI classes $(p=0.0001$ and $p=0.002$, respectively), while no significant changes were observed for resistin. Obese SSc patients also had the highest serum levels of IL-17A $(p=0.01)$, IL-2 $(p=0.001)$ and IL-10 $(p=0.01)$. Adiponectin levels did not significantly change among the BMI subgroups, but the leptin/adiponectin ratio, a functional biomarker of adipose tissue inflammation, was significantly higher in obese patients $(p=0.0001$ ). A striking finding was the increased TNF $\alpha$ levels observed in underweight SSc patients. The latter had significantly higher TNF $\alpha$ levels $(p=0.01)$ than the other BMI classes (Table 3, Figure 2). IL-17A, leptin, and visfatin levels were found to be positively correlated, and TNF $\alpha$ negatively, with increasing BMI values (table 4). BMI was also correlated with ESR, CRP, triglycerides and cholesterol levels (Table 4).

\section{Discussion}

In this study, we investigated the serum levels of different adipokines (adiponectin, leptin, resistin, visfatin) and cytokines (TNF $\alpha$, INF, IL-12, IL-10, IL-17A) in SSc patients and searched for possible correlations with BMI and specific clinical manifestations of the disease. TNF $\alpha$, IL-2, leptin and resistin were higher in SSc patients than in HD. These findings are globally consistent with the literature reporting an increase in cytokines/adipokines in SSc to different extents (4,8,9,16-24). However, attempts to correlate each cytokine/adipokine to the disease activity of SSc and to BMI have yielded conflicting results. All the studies but one (25) showed increased serum levels of leptin in SSc, sometimes correlating with BMI. We found significantly higher leptin levels in SSc than HD and a positive correlation of leptin with BMI, but no correlations with PHA or other clinical manifestations were detected. Instead, a previous study had demonstrated that leptin serum levels were higher in idiopathic PHA and SSc-PAH patients than controls and that dysfunctional endothelial cells from SSC-PAH lung produced leptin "in vitro", although a link with BMI was not investigated (26). Furthermore, we detected significantly higher resistin in SSc patients than in HD, but it was not regulated by BMI, as already reported (6). On the contrary, we found that visfatin levels rose with BMI increases in SSc patients but they were still statistically comparable to HD. Masui et al (22) had detected similar levels of visfatin in SSc patients and controls, but noticed higher visfatin levels in dsSSc patients with late disease, without exploring BMI status. This general inconsistency may be also influenced by treatments, as we found that SSc patients taking PDE5i (tadalafil or sildefanil) but not bosentan, had significantly higher leptin and visfatin levels than patients without PDE5i. Furthermore, SSc-PDE5i patients had 2-3 folds the odds to have high leptin and visfatin levels. It is conceivable that this might be a specific PDE5i effect, rather than related to PAH, as adipokines secretion by white adipocytes is regulated by cAMP and increases upon PDE inhibition "in vitro" (27).

Adiponectin can generally be accounted as leptin antagonist with anti-inflammatory properties and decreases in obesity (28). Adiponectin has also been suggested to have also anti-fibrotic activities and seems to be regulated in SSc, depending on the skin fibrosis extension and disease duration. Some studies demonstrated that adiponectin is low in dcSSc patients both in serum and in lesional skin, but increases in dcSSc patients with a disease duration longer than 5 years, when skin thickness reduces $(9,20,21,29)$. We found low, although not statistically significantly low levels of adiponectin in obese SSc, but we could not confirm previous data as we studied only 15 patients with dcSSc. Interestingly, the ratio leptin/adiponectin was 10-fold higher in obese SSc patients suggesting that the reciprocal leptin/adiponectin regulation is functionally unbalanced in SSc. At this point, a critical question to be addressed is "why is leptin increased in SSc patients as their BMI was lower than in HD"? Indeed, no study has ever demonstrated an increased frequency of obesity among SSc patients. In our cohort, only $6 \%$ had a BMI $>30$ compared to $12.3 \%$ of the general population 
in Apulia (ISTAT, report Osservasalute 2016) implying that leptin overexpression in SSc might be due to some adipocyte dysfunction rather than to an increase production by visceral fat.

Among the investigated cytokines, we found significantly higher levels of TNF $\alpha$ and IL-2 in SSc as compared to HD, presumably linked to the biologic activity of the disease, despite no correlation with the clinical manifestations nor with the global disease activity was found. Within the SSc cohort, obese patients had significantly higher levels of IL-17A and IL-10. A correlation between IL-17A and obesity was expected as high IL-17 mRNA expression has been found in visceral fat of morbidly obese women (30). On the other hand, the significantly higher IL-10 levels in SSc obese patients were unexpected, as in obese subjects IL-10 tends to be low and increases with exercise and weight loss (31). Maybe the most intriguing finding in our analysis was the strikingly high levels of TNF $\alpha$ in underweight SSc patients, roughly 10-folds higher than in normal-weight patients. Increased levels of TNF $\alpha$ in SSc had already been reported $(6,18,19,32)$ although a link with a particular phenotype was not shown. Only one study had demonstrated a correlation of TNF $\alpha$ levels with lung fibrosis and impairment of pulmonary vital capacity (32). Of note, TNF $\alpha$ blocking agents have been successfully used in SSc patients with arthritis (33) and further investigations should focus on this possible pathogenic association. In our study, $10 \%$ of SSc patients were underweight, and loss of body mass has been associated mainly with malabsorption (34). Besides, an overexpression of TNF may also be considered as a further mechanism involved in the chachexia-like status of SSc. During the 1980s, that $\mathrm{TNF} \alpha$ and cachectin were demonstrated to be the two faces of the same coin (35). In an experimental model, $\mathrm{TNF} \alpha$ induced weight loss directly proportional to the decreased food and water intake (36). Moreover, it is known that anti-TNF $\alpha$ drugs may increase body weight and it has been reported that etanercept treatment promoted weight gain and reduced chachexia in patients with rheumatoid arthritis (37).

In conclusion, despite some limitations, such as the cross-sectional design, drug interference, mainly PDE5i, the relatively small sample size of our SSc cohort, this study suggests that an abnormal twist between cytokines, adipokines and BMI takes place in SSc, and these changes in adipokines maybe related to a disfunction of adipocytes (or of other different sources) rather than to the BMI. Further investigation is warranted to establish whether these findings may represent the pathogenetic background of specific clinical manifestations of SSc.

\section{Authors' contributions}

FI and EP conceived the study, were the major participants in its design, coordination, interpretation of results and statistical analysis, they also prepared draft manuscript. DN, RB and NL carried out biological assays, RC, MF and FC collected clinical data and participated in study design coordination. All authors were involved in draft manuscript modifications and approved the final version of the manuscript.

Conflicts of interest: The authors declare no conflict of interest

Data availability statement: Data available on request due to privacy/ethical restrictions.

Funding statement: None

\section{References}

1. Gnacińska M, Małgorzewicz S, Stojek M, Łysiak-Szydłowska W, Sworczak K. Adv Med Sci Role of adipokines in complications related to obesity: a review. 2009 (54):150-7.

2. Iannone F, Fanizzi R, Notarnicola A, Scioscia C, Anelli MG, Lapadula G. Joint Bone Spine Obesity reduces the drug survival of second line biological drugs following a first TNF- $\alpha$ inhibitor in rheumatoid arthritis patients. 2015 (82):187-91.

3. Iannone F, Lopalco G, Rigante D, Orlando I, Cantarini L, Lapadula G. Autoimmun Rev Impact of obesity on the clinical outcome of rheumatologic patients in biotherapy. 2016 (15):447-50.

4. Budulgan M, Dilek B, Dă̆ ŞB, Batmaz I, Yıldız İ, Sarıyıldız MA, et al. . Clin Rheumatol Relationship between serum leptin level and disease activity in patients with systemic sclerosis. 2014 (33):335-9. 
5. Winsz-Szczotka K, Kuźnik-Trocha K, Komosińska-Vassev K, Kucharz E, Kotulska A, Olczyk K. Int J Rheum Dis Relationship between adiponectin, leptin, IGF-1 and total lipid peroxides plasma concentrations in patients with systemic sclerosis: possible role in disease development. 2016 (19):706-14.

6. Pehlivan Y, Onat AM, Ceylan N, Turkbeyler IH, Buyukhatipoglu H, Comez G, et al. . Int J Rheum Dis Serum leptin, resistin and TNF- $\alpha$ levels in patients with systemic sclerosis: the role of adipokines in scleroderma. 2012 (15):374-9.

7. Korman BD, Marangoni RG, Hinchcliff M, Shah SJ, Carns M, Hoffmann A, et al. . Arthritis Rheumatol Brief Report: Association of Elevated Adipsin Levels With Pulmonary Arterial Hypertension in Systemic Sclerosis. 2017 (69):2062-8.

8. Michalska-Jakubus M, Sawicka K, Potembska E, Kowal M, Krasowska D. Postepy Dermatol Alergol Clinical associations of serum leptin and leptin/adiponectin ratio in systemic sclerosis. 2019 (36):325-38.

9. Tomčík M, Arima K, Hulejová H, Kuklová M, Filková M, Braun M, et al. . Cytokine Adiponectin relation to skin changes and dyslipidemia in systemic sclerosis. 2012 (58):165-8.

10. Żółkiewicz J, Stochmal A, Rudnicka L. Arch Dermatol Res The role of adipokines in systemic sclerosis: a missing link? 2019 (311):251-63.

11. van den Hoogen F, Khanna D, Fransen J, Johnson SR, Baron M, Tyndall A, et al. . Arthritis Rheum 2013 classification criteria for systemic sclerosis: an American College of Rheumatology/European League against Rheumatism collaborative initiative. 2013 (65):2737-47.

12. Furst DE, Clements PJ, Steen VD, Medsger TA, Masi AT, D'Angelo WA, et al. . J Rheumatol The modified Rodnan skin score is an accurate reflection of skin biopsy thickness in systemic sclerosis. 1998 (25):84-8.

13. LeRoy EC, Medsger TA. J Rheumatol Criteria for the classification of early systemic sclerosis. 2001 (28):1573-6.

14. Cutolo M, Matucci Cerinic M. Clin Exp Rheumatol Nailfold capillaroscopy and classification criteria for systemic sclerosis. 2007 (25):663-5.

15. Valentini G, Della Rossa A, Bombardieri S, Bencivelli W, Silman AJ, D'Angelo S, et al. . Ann Rheum Dis European multicentre study to define disease activity criteria for systemic sclerosis. II. Identification of disease activity variables and development of preliminary activity indexes. 2001 (60):592-8.

16. Kurasawa K, Hirose K, Sano H, Endo H, Shinkai H, Nawata Y, et al. . Arthritis Rheum Increased interleukin-17 production in patients with systemic sclerosis. 2000 (43):2455-63.

17. Scala E, Pallotta S, Frezzolini A, Abeni D, Barbieri C, Sampogna F, et al. . Clin Exp Immunol Cytokine and chemokine levels in systemic sclerosis: relationship with cutaneous and internal organ involvement. 2004 (138):540-6.

18. Gourh P, Arnett FC, Assassi S, Tan FK, Huang M, Diekman L, et al. . Arthritis Res Ther Plasma cytokine profiles in systemic sclerosis: associations with autoantibody subsets and clinical manifestations. 2009 (11):R147.

19. Hasegawa M, Fujimoto M, Matsushita T, Hamaguchi Y, Takehara K, Sato S. Clin Rheumatol Serum chemokine and cytokine levels as indicators of disease activity in patients with systemic sclerosis. 2011 (30):231-7.

20. Arakawa H, Jinnin M, Muchemwa FC, Makino T, Kajihara I, Makino K, et al. . Exp Dermatol Adiponectin expression is decreased in the involved skin and sera of diffuse cutaneous scleroderma patients. 2011 (20):764-6. 
21. Lakota K, Wei J, Carns M, Hinchcliff M, Lee J, Whitfield ML,et al. . Arthritis Res Ther Levels of adiponectin, a marker for PPAR-gamma activity, correlate with skin fibrosis in systemic sclerosis: potential utility as biomarker? 2012 (14):R102. doi: 10.1186/ar3827.

22. Masui Y, Asano Y, Shibata S, Noda S, Akamata K, Aozasa N, et al. . Rheumatology (Oxford) A possible contribution of visfatin to the resolution of skin sclerosis in patients with diffuse cutaneous systemic sclerosis via a direct anti-fibrotic effect on dermal fibroblasts and Th1 polarization of the immune response. 2013 (52):1239-44.

23. Masui Y, Asano Y, Akamata K, Aozasa N, Noda S, Taniguchi T, et al. . Rheumatol Int Serum resistin levels: a possible correlation with pulmonary vascular involvement in patients with systemic sclerosis. 2014 (34):1165-70.

24. Neumann E, Lepper N, Vasile M, Riccieri V, Peters M, Meier F, et al. . Cytokine Adipokine expression in systemic sclerosis lung and gastrointestinal organ involvement. 2019 (117):41-9.

25. Kotulska A, Kucharz EJ, Brzezińska-Wcisło L, Wadas U. Clin Rheumatol A decreased serum leptin level in patients with systemic sclerosis. 2001 (20):300-2.

26. Huertas A, Tu L, Gambaryan N, Girerd B, Perros F, Montani D, et al. . Eur Respir J Leptin and regulatory T-lymphocytes in idiopathic pulmonary arterial hypertension. 2012 (40):895-904.

27. Komai AM, Brännmark C, Musovic S, Olofsson CS. J Physiol PKA-independent cAMP stimulation of white adipocyte exocytosis and adipokine secretion: modulations by Ca2+ and ATP. 2014 (592):5169-86.

28. Tilg H, Moschen AR. Clin Sci (Lond) Role of adiponectin and PBEF/visfatin as regulators of inflammation: involvement in obesity-associated diseases. 2008 (114):275-88.

29. Masui Y, Asano Y, Shibata S, Noda S, Aozasa N, Akamata K, et al. . J Eur Acad Dermatol Venereol Serum adiponectin levels inversely correlate with the activity of progressive skin sclerosis in patients with diffuse cutaneous systemic sclerosis. 2012 (26):354-60.

30. Zapata-Gonzalez F, Auguet T, Aragonès G, Guiu-Jurado E, Berlanga A, Martinez S, et al. . Int J Mol Sci Interleukin-17A Gene Expression in Morbidly Obese Women. 2015 (16):17469-81.

31. Dorneles GP, Haddad DO, Fagundes VO, Vargas BK, Kloecker A, Romão PR, et al. . Cytokine High intensity interval exercise decreases IL- 8 and enhances the immunomodulatory cytokine interleukin-10 in lean and overweight-obese individuals. 2016 (77):1-9.

32. Hasegawa M, Fujimoto M, Kikuchi K, Takehara K. J Rheumatol Elevated serum tumor necrosis factoralpha levels in patients with systemic sclerosis: association with pulmonary fibrosis. 1997 (24):663-5.

33. Distler JH, Jordan S, Airo P, Alegre-Sancho JJ, Allanore Y, Balbir Gurman A, et al. . Clin Exp Rheumatol Is there a role for $\mathrm{TNF} \alpha$ antagonists in the treatment of SSc? EUSTAR expert consensus development using the Delphi technique. 2011 (29):S40-5.

34. Bishop V, Harrison E, Lal S, Herrick AL. Scand J Rheumatol Evidence for a clinical association between body mass index and malabsorption in patients with systemic sclerosis. 2015 (44):341-3.

35. Beutler B, Greenwald D, Hulmes JD, Chang M, Pan YC, Mathison J, et al. . Nature Identity of tumour necrosis factor and the macrophage-secreted factor cachectin. 1985 (316):552-4.

36. Mahony SM, Beck SA, Tisdale MJ. Br J Cancer Comparison of weight loss induced by recombinant tumour necrosis factor with that produced by a cachexia-inducing tumour. 1988 (57):385-9.

37. Chen CY, Tsai CY, Lee PC, Lee SD. Curr Pharm Des Long-term etanercept therapy favors weight gain and ameliorates cachexia in rheumatoid arthritis patients: roles of gut hormones and leptin. 2013 (19):1956-64.

Table 1. Clinical findings of Systemic Sclerosis (SSc) cohort (n. 89 patients). 


\begin{tabular}{ll}
\hline Disease duration (years), mean \pm SD & $8.1 \pm 6.6$ \\
\hline Underweight (BMI [?]18.5), n. (\%) & $9(10 \%)$ \\
Normal weight (BMI >8.5 <25), n. (\%) & $49(55 \%)$ \\
Overweight (BMI [?]25 [?]30), n. (\%) & $26(29 \%)$ \\
Obese (BMI >30), n. (\%) & $5(6 \%)$ \\
Diabetes Mellitus, n. (\%) & $1(9 \%)$ \\
Dyslipidemia, n. (\%) & $11(12 \%)$ \\
Tot-Cholesterol/HDL, mean \pm SD & $3.3 \pm 1.0$ \\
Framingham risk score, mean \pm SD & $3.7 \pm 2.9$ \\
Previous smokers, n (\%) & $5(6 \%)$ \\
Arterial Hypertension, n (\%) & $8(7 \%)$ \\
Active patients with disease activity index [?]3 ESSG, n. (\%) & $24(27 \%)$ \\
Erythrocyte Sedimentation Rate (mm/h), mean \pm SD & $19 \pm 14$ \\
C-Reactive Protein (mg/l), mean \pm SD & $4.3 \pm 5$ \\
Limited cutaneous SSc, n. (\%) & $74(83 \%)$ \\
Interstitial lung disease, n. (\%) & $51(57 \%)$ \\
Esophageal Involvement, n. (\%) & $79(89 \%)$ \\
Bowel incontinence, n. (\%) & $2(3 \%)$ \\
Active digital ulcers, n. (\%) & $18(20 \%)$ \\
Digital pitting scars, n. (\%) & $55(62 \%)$ \\
Calcinosis, n. (\%) & $79(89 \%)$ \\
Renal Involvement, n. (\%) & $5(6 \%)$ \\
Pulmonary Arterial Hypertension, n. (\%) & $7(8 \%)$ \\
Capillaroscopy scleroderma pattern, n. (\%) & Early $6(7 \%)$ \\
& Active $49(55 \%)$ \\
& Late $34(38 \%)$ \\
\hline
\end{tabular}

Table 2. Demographics and cytokines/adipokines serum levels in Systemic Sclerosis (SSc) patients as whole cohort and

subdivided according to phosphodiesterase-5 (PDE5) inhibitors (tadalafil/sildefanil) intake, and healthy donors (HD).

Data are shown as mean \pm standard deviation.

\begin{tabular}{|c|c|c|c|c|c|c|}
\hline & $\begin{array}{l}\text { Comparison } \\
\text { SSc-all vs } \\
\text { healthy } \\
\text { donors (HD) }\end{array}$ & $\begin{array}{l}\text { Comparison } \\
\text { SSc-all vs } \\
\text { healthy } \\
\text { donors (HD) }\end{array}$ & $\begin{array}{l}\text { Comparison } \\
\text { SSc-all vs } \\
\text { healthy } \\
\text { donors (HD) }\end{array}$ & $\begin{array}{l}\text { Comparison } \\
\text { SSc } \\
\text { tadalafil/sildefani } \\
\text { vs SSc other } \\
\text { drugs (OD) }\end{array}$ & $\begin{array}{l}\text { Comparison } \\
\text { SSc } \\
\text { iltadalafil/sildefan } \\
\text { vs SSc other } \\
\text { drugs (OD) }\end{array}$ & $\begin{array}{l}\text { Comparison } \\
\text { SSc } \\
\text { iiltadalafil/sildefan } \\
\text { vs SSc other } \\
\text { drugs (OD) }\end{array}$ \\
\hline & SSc all (n. 89) & HD (n. 26) & $\mathrm{p}$ & $\begin{array}{l}\text { SSc PDE5 } \\
\text { inhibitors (n. } \\
8 \text { ) }\end{array}$ & SSc OD (81) & $\mathrm{p}$ \\
\hline Age (years) & $52.1 \pm 14$ & $49.4 \pm 11$ & 0.07 & $64.5 \pm 14$ & $50.3 \pm 13$ & 0.0001 \\
\hline $\begin{array}{l}\text { Female } \mathrm{n} \\
(\%)\end{array}$ & $84(94 \%)$ & $23(88 \%)$ & 0.37 & $7(87 \%)$ & $76(93 \%)$ & 0.37 \\
\hline $\begin{array}{l}\text { Waist cir- } \\
\text { cumference } \\
(\mathrm{cm})\end{array}$ & $80.7 \pm 9.5$ & $88.4 \pm 12$ & 0.49 & $90 \pm 5.6$ & $79.6 \pm 9.4$ & 0.06 \\
\hline
\end{tabular}




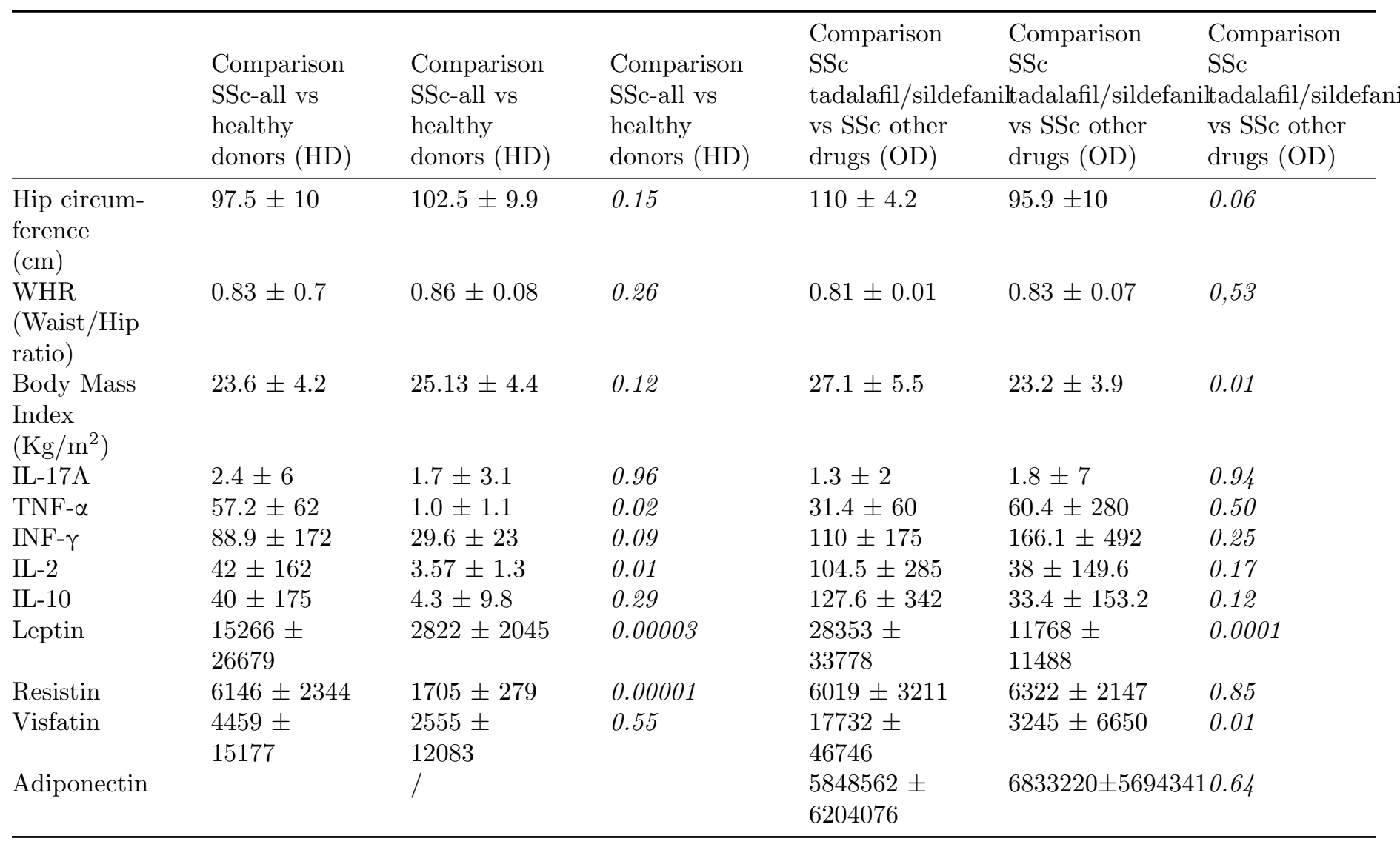

Table 3. Cytokines and adipokines in Systemic Sclerosis patients by BMI categories (mean \pm SD).

\begin{tabular}{|c|c|c|c|c|c|}
\hline & Underweight & Normal-weight & Overweight & Obese & $p$ \\
\hline $\mathrm{INF}_{\gamma}$ & $76.4 \pm 125$ & $83.7 \pm 251$ & $105.0 \pm 303$ & $91.5 \pm 249$ & 0.97 \\
\hline IL-2 & $23.4 \pm 85$ & $11.4 \pm 45$ & $35.9 \pm 129$ & $\begin{array}{l}233 \pm 350 \text { vs: } \\
U W, N W \text { OW }\end{array}$ & 0.001 \\
\hline IL-10 & $12.0 \pm 31$ & $12.8 \pm 35$ & $30.1 \pm 112$ & $\begin{array}{l}204 \pm 431 \text { vs: } \\
\text { UW, NW OW }\end{array}$ & 0.01 \\
\hline Leptin & $9033 \pm 7181$ & $9623 \pm 14097$ & $17465 \pm 10335$ & $\begin{array}{l}64970 \pm 89346 \\
\text { vs: } U W, N W \\
O W\end{array}$ & 0.0001 \\
\hline Adiponectin & $\begin{array}{l}9829075 \pm \\
66275\end{array}$ & $\begin{array}{l}6582923 \pm \\
53445\end{array}$ & $\begin{array}{l}7459125 \pm \\
65585\end{array}$ & $\begin{array}{l}3248520 \pm \\
10425\end{array}$ & 0.22 \\
\hline
\end{tabular}




\begin{tabular}{llllll}
\hline & Underweight & Normal-weight & Overweight & Obese & $p$ \\
\hline Leptin/Adiponec & $0.002 \pm 0.002$ & \multirow{2}{*}{$0.002 \pm 0.004$} & \multirow{2}{*}{$0.003 \pm 0.0006$} & $0.02 \pm 0.04$ vs: & \multirow{2}{*}{0.0001} \\
& & & UW, NW OW & \\
\hline
\end{tabular}

$\mathrm{UW}=$ Underweight, $\mathrm{NW}=$ Normal-weight, $\mathrm{OW}=$ Overweight, $\mathrm{Ob}=$ Obese.

Table 4. Correlation of cytokines/adipokines with anthropometric and clinical findings. Data are shown as mean \pm SD. Only the significant correlations are reported.

\begin{tabular}{lllllllll}
\hline & & & & & Chol & & \\
& IL-17A & Leptin & Visfatin & TNF $\alpha$ & Trigl & LDL & ESR & CRP \\
\hline BMI & $r 0.25 p$ & $r 0.41 p$ & $r 0.25 p$ & $r-0.27 p$ & $r 0.28 p$ & $r 0.46 p$ & $r 0.30 p$ & $r 0.32$ \\
& 0.02 & 0.0001 & 0.02 & 0.05 & 0.02 & 0.002 & 0.007 & 0.004 \\
\hline
\end{tabular}

$\mathrm{BMI}=$ Body, Mass, Index, Trigl $=$ Triglycerides, $\mathrm{Chol}=$ Cholesterol, ESR $=$ Erythrocyte Sedimentation Rate, $\mathrm{CRP}=\mathrm{C}$-reactive protein, $\mathrm{r}=$ coefficient correlation, $\mathrm{p}=$ significance level

\section{Legends}

Figure 1. Serum leptin levels in healthy donors (HD) and Systemic Sclerosis (SSc) patients with different categories of body mass index. (U-W: under-weight, N-W normal-weight, O-W over-weight). Mean (95\% CI)

Figure 2. Serum TNF $\alpha$ levels in healthy donors (HD) and Systemic Sclerosis (SSc) patients with different categories of body mass index. (U-W: under-weight, N-W normal-weight, O-W over-weight). Mean (95\% CI)

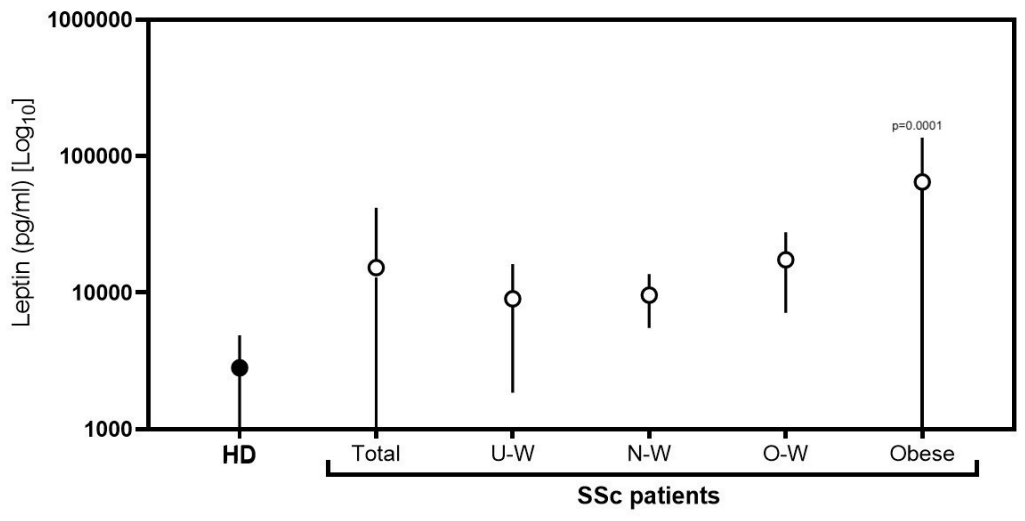




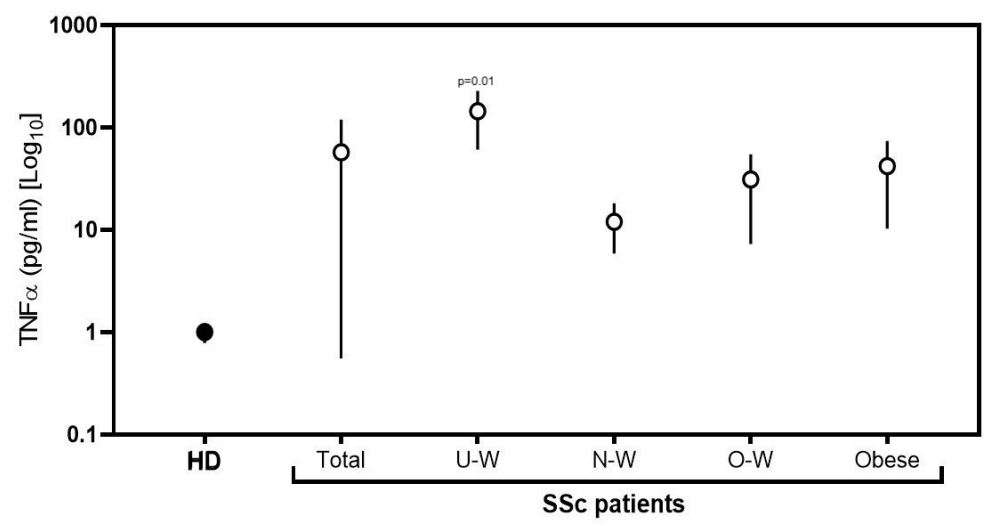

